\section{A ENDOTHELIAL CELL NITRIC OXIDE BIOAVAILABILITY AND INSULIN SENSITIVITY ARE REGULATED BY IGF-1 AND INSULIN RECEPTOR LEVELS}

doi:10.1136/heartjnl-2011-300110.1

A Abbas, H Viswambharan, H Imrie, A Rajwani, M Kahn, M Gage, R Cubbon, J Surr, S Wheatcroft, M Kearney. Leeds Institute of Genetics Health and Therapeutics, Leeds, UK

Background In a similar manner to insulin, the growth promoting hormone Insulin-like Growth Factor-1 (IGF-1), may be an important regulator of endothelial nitric oxide (NO) bioavailability. We have previously reported evidence of increased basal NO production in the vasculature in two murine models of reduced IGF-1 receptor (global hemizygous knockout (IGFRKO) and endothelial cell specific IGF-1R knockout (ECIGFRKO)). Augmentation of this increase in $\mathrm{NO}$ is relative to progressive decrease in IGF-1R number (WT vs ECIGFRKO hemizygotes $p=0.01$, WT vs ECIGFRKO homozygotes $\mathrm{p}=0.001$ ). Furthermore, by decreasing IGF-1R numbers in the insulin resistant hemizygous insulin receptor knockout (IRKO) model (IRKO $\times$ IGFRKO) we have shown insulin sensitivity in the vasculature can be restored. In this study, we have investigated further these receptor interactions with the generation of a mouse overexpressing the human IGF-1R specifically in the endothelium under control of the Tie-2 promoter-enhancer (hIGFREO), and by targeted knockdown of the IGF-1R in human umbilical vein endothelial cells (HUVECs).

Methods Metabolic function was assessed in mice by tolerance tests using whole-blood micro-sampling after insulin or glucose intraperitoneal injection. Cardiovascular function was assessed by thoracic aortic vasomotion ex vivo in the organbath. Complimentary in vitro studies were conducted by siRNA mediated downregulation of the IGF-1 receptor in HUVECs with and wihout insulin stimulation. Nitric oxide synthase activity was measured using an assay measuring conversion of [14C]-L-arginine to [14 C]-L-citrulline.

Results Glucose and insulin tolerance testing showed no difference between hIGFREO mice and wild-type (WT) littermates. Murine thoracic aorta from hIGFREO mice were hypercontractile to phenylepherine (PE) compared to WT (Emax hIGFREO= $0.91 \pm 0.045 \mathrm{~g} ; \quad \mathrm{WT}=0.62 \pm 0.045 \mathrm{~g}, \quad \mathrm{p}=0.0036)$ with decreased response to LNMMA (Emax hIGFREO $=47.70 \pm 9.87 \mathrm{~g}$; $W T=106.1 \pm 30.10 \mathrm{~g}, p=0.048)$. These data indicate reduced endothelial NO bioavailability in hIGFREO mice compared to WT. HUVECs transfected with IGF1R-siRNA showed increased basal and insulin mediated eNOS phosphorylation in the presence of insulin (Ins: $164 \pm 4.9 \%$ vs siRNA+Ins: $192 \pm 0.7 \%, p<0.05$ ). eNOS activity (L-arginine, L-citrulline assay) was enhanced upon trans-
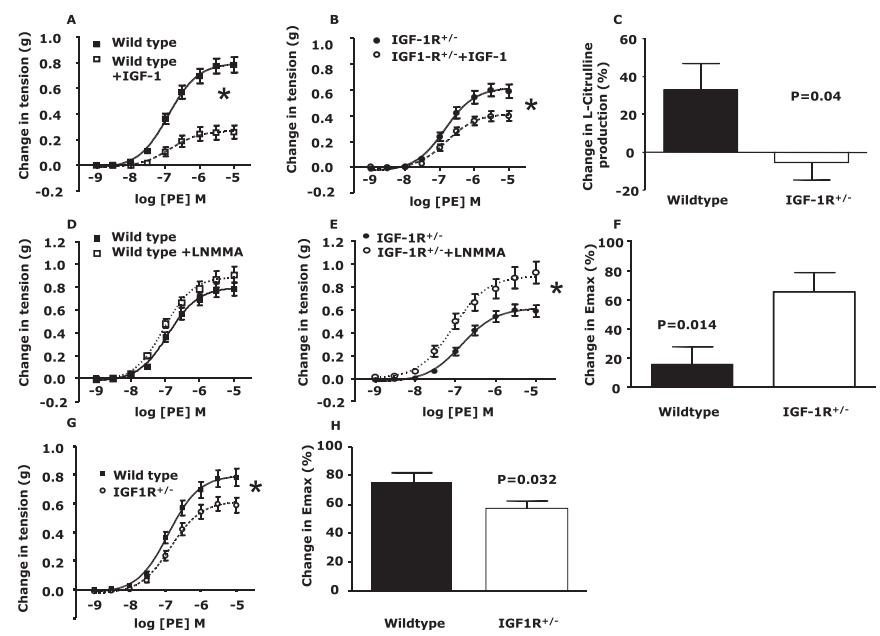

Abstract A Figure 1
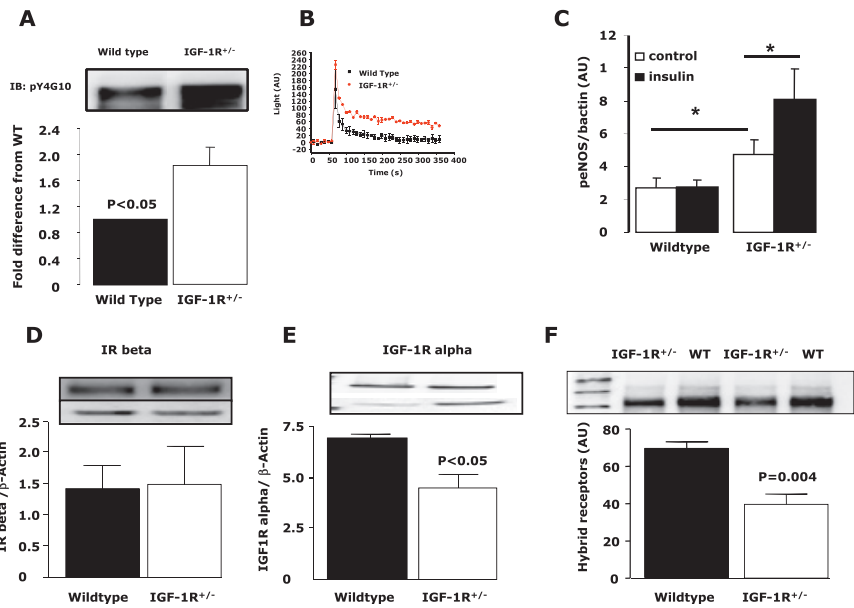

Abstract A Figure 2
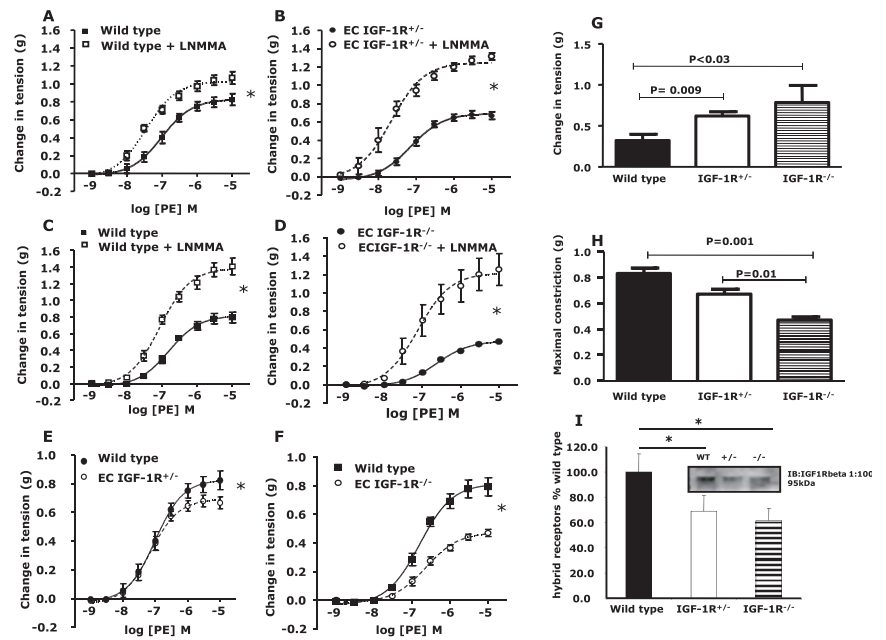

Abstract A Figure 3
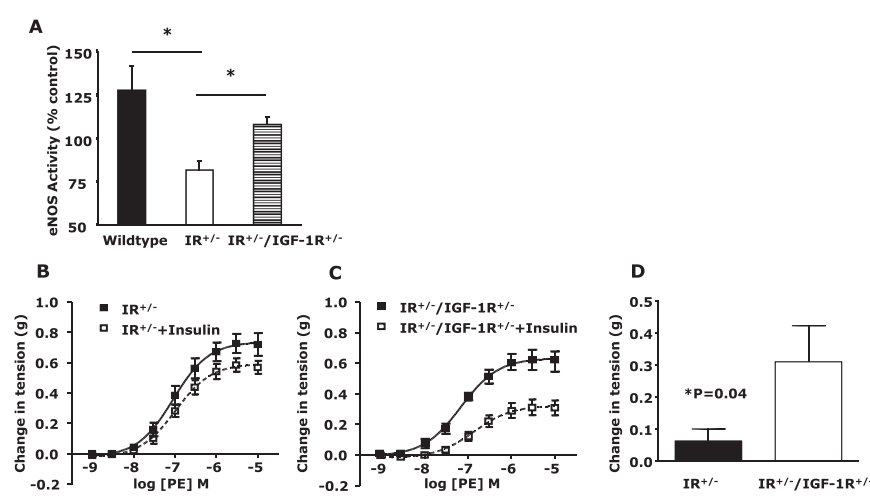

Abstract A Figure 4

fection with IGF1R-siRNA (Scrambled siRNA: $95.7 \pm 13.7 \%$ vs IGF1R-siRNA: $188.7 \pm 48.3 \%, \mathrm{p}<0.05)$.

Implications These data demonstrate that increasing numbers of IGF-1R specifically in murine endothelium leads to reduced NO bioavailability. Complementary siRNA studies confirm results of previous murine studies that reducing IGF-1R numbers enhance NO bioavailability. Therefore this raises the intriguing possibility that manipulation of IGF-1R numbers may represent a novel therapeutic 


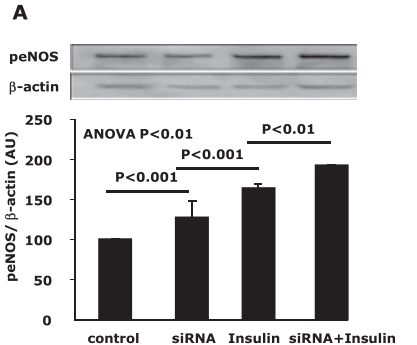

B

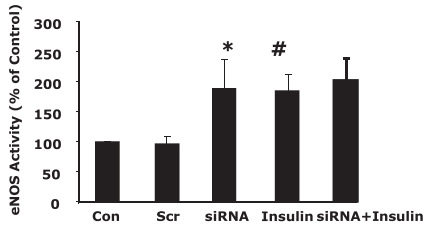

Abstract A Figure 5
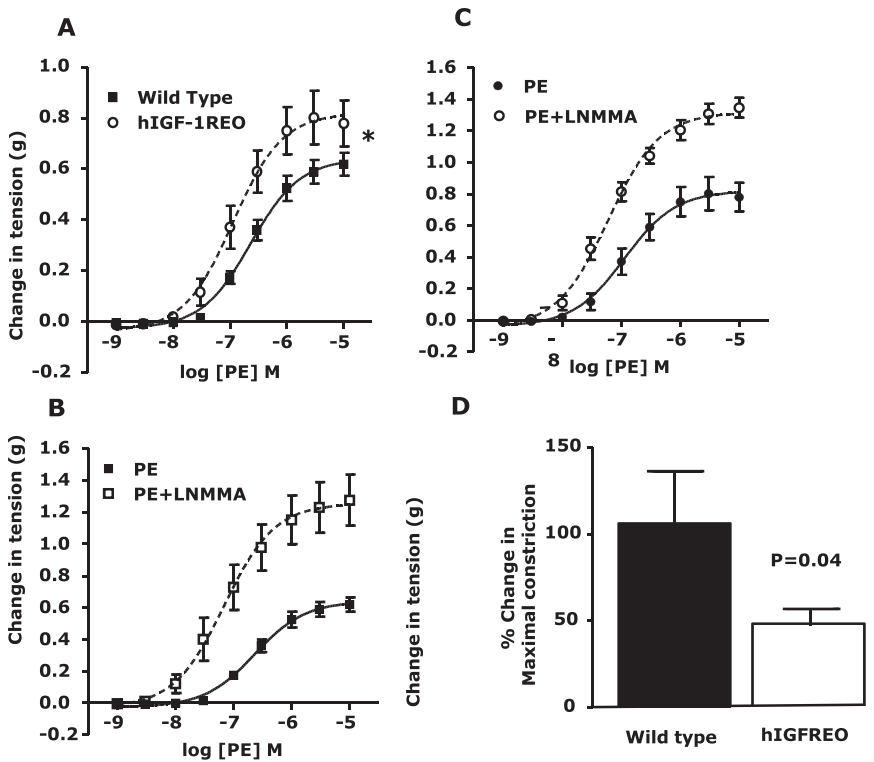

Abstract A Figure 6

strategy by which to modify vascular NO bioavailability and endothelial cell insulin sensitivity.

\section{B VH-IVUS FINDINGS PREDICT MAJOR ADVERSE CARDIOVASCULAR EVENTS. THE VIVA STUDY (VIRTUAL HISTOLOGY INTRAVASCULAR ULTRASOUND IN VULNERABLE ATHEROSCLEROSIS)}

doi:10.1136/heartjnl-2011-300110.2

${ }^{1} \mathrm{P}$ A Calvert, ${ }^{1} \mathrm{D}$ R Obaid, ${ }^{2} \mathrm{~N}$ E J West, ${ }^{2} \mathrm{~L}$ M Shapiro, ${ }^{2} \mathrm{D}$ McNab, ${ }^{2} \mathrm{C} \mathrm{G}$ Densem, ${ }^{2} \mathrm{P}$ M Schofield, ${ }^{2} \mathrm{D}$ Braganza, ${ }^{2} \mathrm{~S} \mathrm{C}$ Clarke, ${ }^{2} \mathrm{M}$ O'Sullivan, ${ }^{3} \mathrm{~K}$ K Ray, ${ }^{1} \mathrm{M}$ R Bennett. ${ }^{1}$ University of Cambridge, Cambridge, UK; ${ }^{2}$ Papworth Hospital NHS Foundation Trust, Cambridge, UK: ${ }^{3}$ St George's University of London, London, UK

Background Identification of high-risk atherosclerotic plaques offers opportunities for risk stratification and targeted intensive treatment of patients with coronary artery disease. Virtual Histology intravascular ultrasound (VH-IVUS) has been validated in human atherectomy and post-mortem studies and can classify plaques into presumed high- and low-risk groups. However, VH-IVUS-defined plaques have not been shown to be associated with major adverse cardiovascular events (MACE), or biomarkers that confer increased cardiovascular risk, such as serum cytokines or shortened leukocyte telomere length (LTL)

Methods 170 patients with stable angina or troponin-positive acute coronary syndrome (ACS), referred for percutaneous coronary intervention (PCI) were prospectively enrolled and underwent full 3-vessel VH-IVUS pre-PCI. Troponin-I (cTnI), IL-6, IL-18, hsCRP, neopterin, MCP-1 and sICAM-1 were measured pre-PCI and 24-h post-PCI. LTL was determined by qPCR. The combined primary endpoint (MACE) included unplanned revascularisation, myocardial infarction (MI) and death, with a secondary endpoint of post-PCI MI (MI 4a).

Results 18 MACE occurred in 16 patients (median follow-up: 625 (463-990) days). $30372 \mathrm{~mm}$ of VH-IVUS were analysed and 1106 plaques classified (Abstract B Figure 1) locally and via a core-lab. After multivariable regression:

1. Total number of non-calcified VH-IVUS-identified thin capped fibroatheromata (VHTCFA) was the only factor independently associated with MACE ( $\mathrm{HR}=3.16$, (95\% $\mathrm{CI}=1.16$ to 8.64$), \mathrm{p}=0.025)$.

2. Total VHTCFA number $(\mathrm{OR}=1.26(1.03$ to 1.53$) \mathrm{p}=0.021)$ and total stent length $(\mathrm{OR}=1.04$ (1.01 to 1.08$), \mathrm{p}=0.01)$ were the only factors independently associated with MI 4a.

3. A novel 3-vessel vulnerability index (necrotic core: fibrous tissue ratio) and side branch loss were independently associated with stenting-related $\mathrm{cTnI}$ rise (standardised beta coefficient $(s \beta)=0.29, p=0.004$ and $s \beta=0.23, p=0.019$ respectively).

4. Necrotic core area at the minimum luminal area frame was the only factor independently associated with ACS presentation $(\mathrm{OR}=1.59, \mathrm{p}=0.030)$.

5. Stented vessel VHTCFA number $(\mathrm{OR}=1.75$ (1.22 to 2.51), $\mathrm{p}=0.002)$ was independently associated with the lower LTL tertile (DNA-based cardiovascular risk predictor).

6. Stenting-related IL-6 rise was the only biomarker independently associated with MACE ( $\mathrm{HR}=1.03 \quad(1.01-1.05)$, $\mathrm{p}=0.007$ ).

Conclusion We present the first report of an association between VHTCFA and MACE. This provides novel evidence that VHTCFA definitions are important in their own right (rather than as analogues of histological TFCA definitions). We also present the first report of associations between VHTCFA and MI $4 \mathrm{a}$ as well as a novel vulnerability index that is association with stenting-related troponin rise. Finally, we report a novel association between VHTCFA and DNA-based cardiovascular risk prediction (LTL)

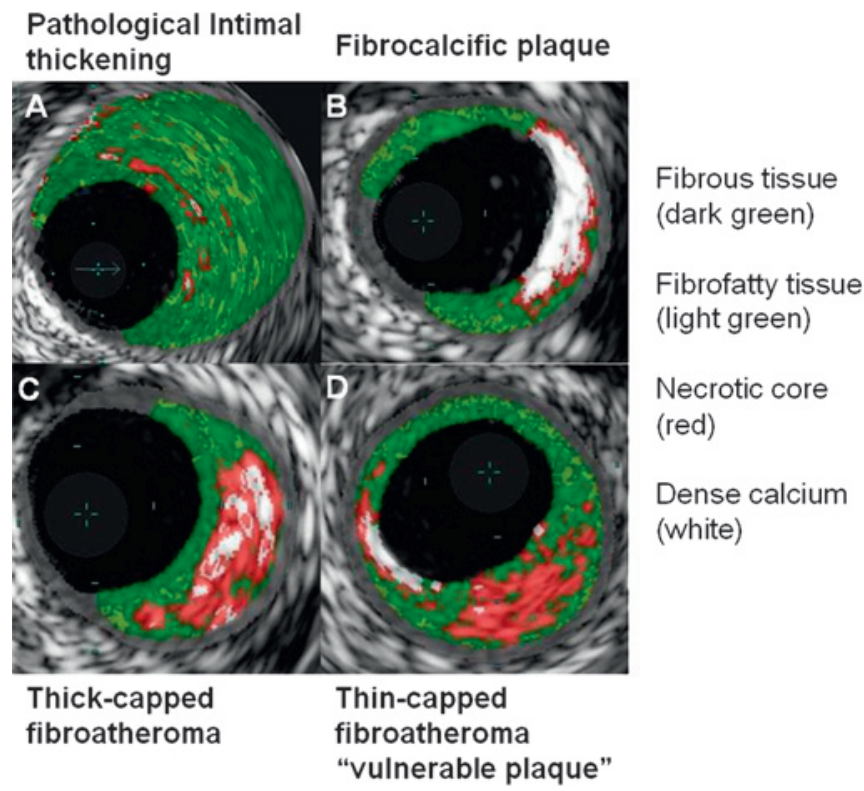

Abstract B Figure 1 